\title{
Desarrollo de control e implementación de robot móvil con la capacidad de solucionar laberintos complejos
}

Diego Armando Roa Ramos*

Brayan Andrés Báez Ruiz*

Daniela Alejandra Mosquera Osuna***

\section{Resumen}

Este proyecto se dividió en 4 etapas. En la primera se realizó un estudio de los módulos experimentales de algunos convertidores de frecuencia. En la segunda se diseñó el convertidor y se hicieron los cálculos respectivos. En la tercera se construyó el módulo experimental (construcción del diseño del convertidor frecuencia-voltaje al aplicar un generador y un encoder que permiten el análisis de RMP de un motor). En la cuarta se analizaron los valores teóricos y prácticos del motor para su validación y determinar su ecuación de transferencia.

Palabras clave: controlador PID, encoder, función de transferencia, laberinto, robot, robótica móvil, señal

\begin{abstract}
This project was divided into 4 stages. In the first one a study of the experimental modules of some frequency converters is carried out. In the second the converter was designed and the respective calculations were made. In the third one the experimental module was built (construction of the design of the frequency-voltage converter by applying a generator and an encoder that allow the RPM analysis of an engine). In the last one, the theoretical and practical values of the motor were analyzed for validation and to determine its transfer equation.
\end{abstract}

Keywords: Encoder, Mobile Robotics, PID Controller, Robot Maze, Signal, Transfer Function

\footnotetext{
* Estudiante de la Corporación Unificada Nacional de educación superior (CUN). Contacto: diego.roa@cun.edu.co

** Estudiante de la Corporación Unificada Nacional de educación superior (CUN). Contacto: brayan.baez@cun.edu.co

${ }^{* * *}$ Estudiante de la Corporación Unificada Nacional de educación superior (CUN). Contacto: daniela.mosquera@cun.edu.co
} 


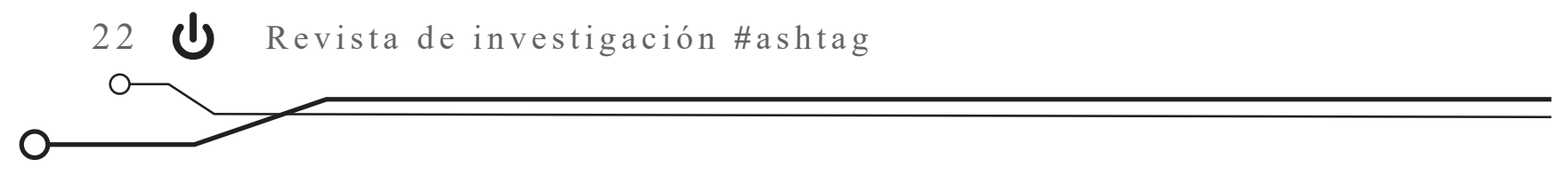

\section{Introducción}

En este artículo se tendrá como foco el desarrollo desde cero de un robot móvil con la capacidad de superar laberintos de alta complejidad, cuyo fin es servir de base para futuros proyectos. El objetivo principal es diseñarlo para participar en un concurso que se lleva a cabo en las instalaciones de la Corporación Unificada de Educación Superior (CUN), en el que varios grupos conformados por estudiantes presentan sus robots móviles modificados y adaptados para superar diferentes retos. Uno de los retos opcionales es el del laberinto; este supone poder controlar de manera manual o autónoma al robot -por supuesto, la segunda opción otorga más puntaje-. Sin embargo, son muy pocos los grupos que implementan un sistema autónomo por su complejidad y configuración avanzada de sensores.

Como mencionan Bueno y Ríos (2008), la robótica móvil hoy en día es considerada una tecnología útil que facilita la solución de problemáticas en diferentes entornos, por ejemplo, la solución del reto laberinto de forma autónoma. Una de las desventajas que presenta nuestro proyecto es que el sistema de navegación autónoma no está basado en sistemas difusos; sin embargo, los sensores que implementamos dan una buena visibilidad de los obstáculos, lo que permite desarrollar funcionalidades más simples.

Bonilla et al. (2009) mencionan la necesidad de incrementar la autonomía en las aplicaciones robóticas. Arguyen esto como una motivación para la creación y el desarrollo de robots móviles cuyo afán es limitar lo más posible la intervención humana. Ahora bien, una de las desventajas que percibimos es que no contamos con una cámara web y un sistema de control difuso, lo que hace menos funcional nuestro robot móvil. En este documento también se presenta una solución para la detección de obstáculos por parte de los sensores que evita realizar pruebas para identificarlo. Por su parte, Mendoza (2013) muestra el diseño y la construcción de un robot con la capacidad de recoger bolas y a su vez solucionar un laberinto. Con base en este artículo se descartó el funcionamiento de los sensores CNY70, ya que presentan un margen de error más alto y un retraso en su respuesta.

Los planteamientos de Bambino (2008), proponen varios aspectos importantes y relevantes frente al diseño, implementación y desarrollo matemático de robots móviles que esquivan obstáculos. No obstante, como desventaja se identificó que la programación se orienta en Python y nosotros no la manejamos.

Rodríguez y Reyes (2001) proponen una solución para implementar en un robot móvil la detección y evasión de obstáculos en un ambiente desconocido. Este artículo resultó de gran importancia para la investigación porque explica una iniciativa que utiliza una programación similar a la nuestra y va en torno a PICC, que manejamos al momento de implementar nuestro código para el desarrollo del software que controlara nuestro robot móvil. 


\section{Planteamiento del problema}

¿Cómo superar la prueba del laberinto, reto CUN, con un sistema de control autónomo?

\section{Antecedentes}

La automatización, implementación y fabricación de robots móviles son actividades que han crecido de forma significativa. Cada vez es más fácil encontrarlas en las tareas más complicadas y riesgosas para las personas, ocasiones en donde se intenta que un robot las realice. Los robots de tipo terrestre como vehículos seguidores de línea, detectores de colores, autónomos, detectores de obstáculos, entre otros, así como sus diseños y funciones se han venido desarrollando y muchos de ellos son usados hoy en día para tareas diversas.

\section{Laberinto}

Este reto está diseñado para que el equipo lo resuelva de forma autónoma. El robot se ubicará en el inicio del laberinto, en la zona verde, donde iniciará el recorrido. El operador accionará el

\section{Marco teórico}

En el mercado existen robots con características similares a las de los siguientes aparatos.

\section{Aspiradora robot}

Es un robot diseñado para limpieza del hogar en ausencia de sus habitantes. Presta servicios
Actualmente encontramos que tanto la ingeniería electrónica como la mecánica en conjunto resuelven problemas que vemos diariamente a nuestro alrededor. Por lo tanto, este desarrollo surge ante la problemática establecida para crear e implementar un robot móvil con capacidad autónoma para detectar y esquivar obstáculos que se presenten en su camino y que tenga como referencia una distancia aproximada de $6 \mathrm{~cm}$ a la pared.

robot y no podrá manipular el robot hasta que este llegue al final sin tocar ninguna de las paredes, como indica el reto.

avanzados que permiten su puesta en marcha cuando el usuario no se encuentra en el domicilio. 


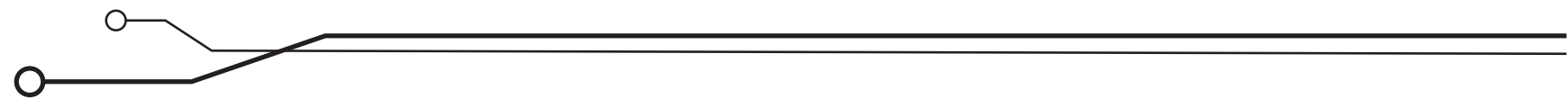

Figura 1. Aspiradora robot

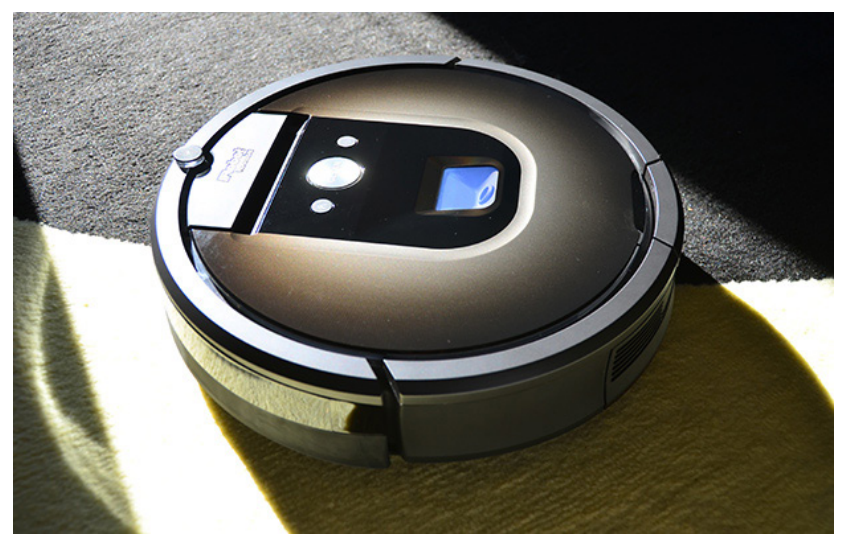

Fuente: Ackerman, E. (2015)

Características:

Autonomía: Aprox. 2 horas

Tiempo de Carga: Aprox. 2 horas

Navegación Autónoma: Sí

\section{Casas automatizadas}

Es una unión entre arquitectura, diseño y tecnología muy avanzada que da paso a la automatización. En este momento se está muy cerca de
Modelo: iRobot Roomba 980

Capacidad: 0,6 lt

Inalámbrica: Sí

Percepción: Cámara abordo

Figura 2. Casa inteligente

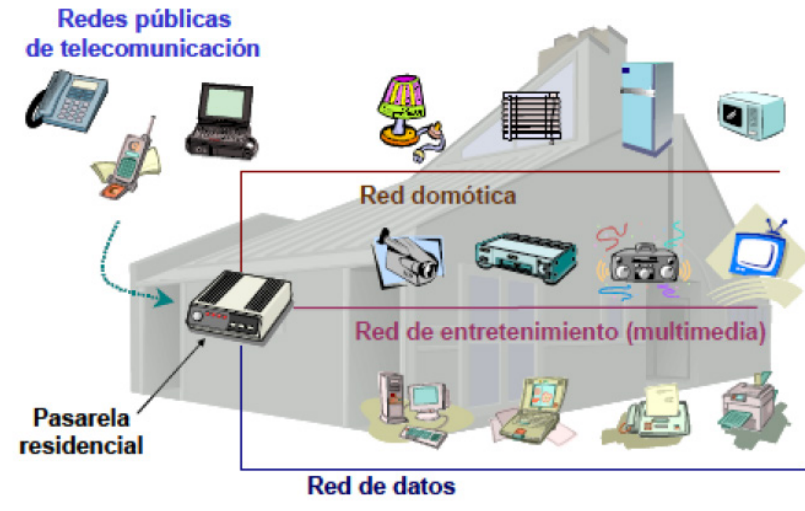

Fuente: Millán, R. (2004)

Características: video cinema, audio inteligente, cortinas automáticas, luces dimerizables e inteligentes, asistente 24/7. 


\section{Unidades de seguridad}

Es un robot autónomo capaz de trabajar en ambientes reales. La compañía Aethon fabricó un robot llamado Tug, creado para realizar tareas básicas como entrega de comida, medicamentos y hacer recorridos en un hospital. Esto consigue que el personal médico pueda dedicarse a tareas más importantes.

Figura 3. Unidades de seguridad

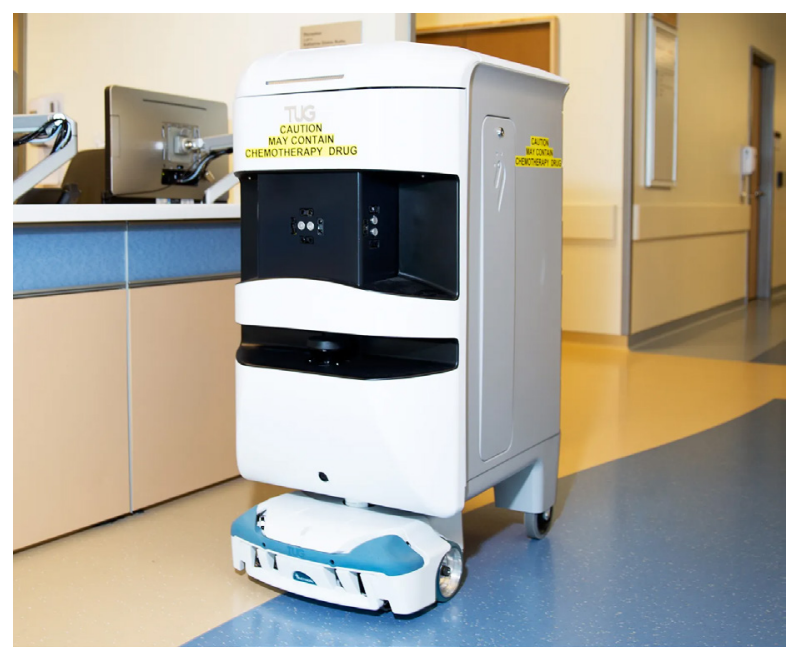

Fuente: Simon, M. (2015)

\section{Brazo robótico}

Es un robot móvil y se utiliza en empresas de producción y en logística de almacén, espacios donde se pueden automatizar tareas. Algunos de estos se pueden trasladar hacia atrás y hacia adelante.

Figura 4. Brazo robótico

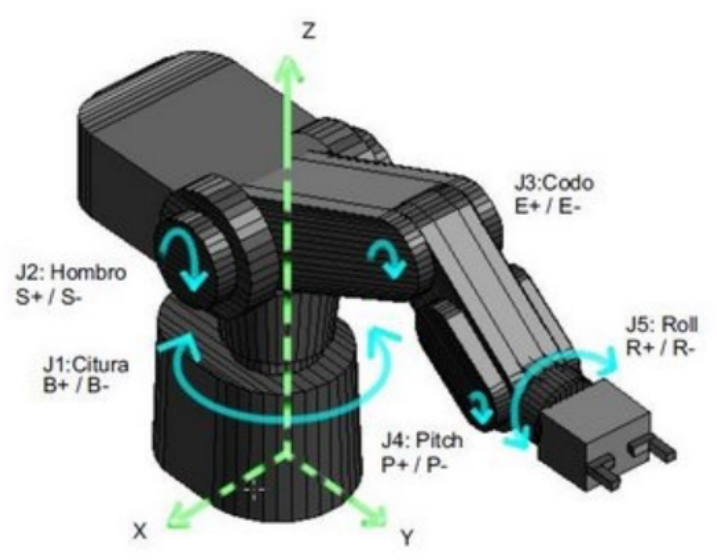

Fuente: Berenz, G. (2008) 


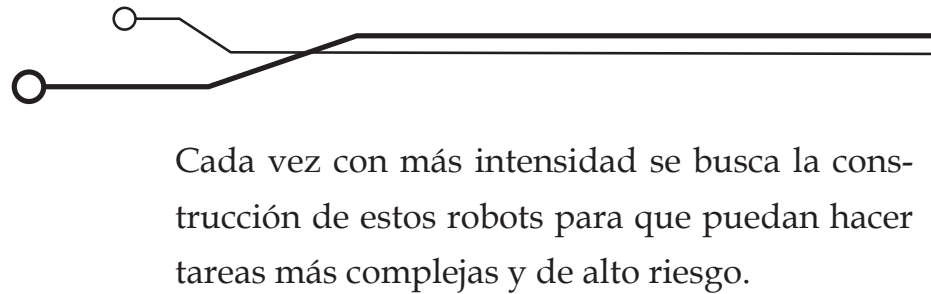

\section{Solución propuesta}

En el desarrollo del proyecto se consultaron diferentes tipos de documentos que muestran resultados obtenidos a través de varios métodos y sensores que resuelven problemas similares al propuesto. Por esta razón, se decantó del conjunto un modo de obtener el mejor resultado de respuesta para nuestro robot móvil

Paso 1. Se diseña el robot con el tamaño adecuado para su participación en la prueba.

Para el chasis del robot móvil se eligió la impresión en madera MDF, tras considerar que el peso y el grosor de este material permiten una mejor movilidad y garantizan, por ende, un menor esfuerzo de los motores.

1.1. Se diseñan los planos del robot móvil con las medidas especificadas por el creador del reto laberinto.
1.2. Se fabrica cada una de las piezas que se utilizarán en el robot móvil.

1.3. Se ensambla el robot y se realizan pruebas de movimiento.

Paso 2. Calibración y selección de sensor.

2.1. Se eligió usar un sensor de ultrasonido por su capacidad de identificar los obstáculos puestos en el transcurso del laberinto, siempre y cuando se mantenga a una distancia de $6 \mathrm{~cm}$ de la pared, ya que una distancia mayor hace que se presenten errores en la conversión del dato. Cai y Regtien presentan un método de medición del tiempo de vuelo basado en la detección de la amplitud nula de la envolvente. El proceso consiste en generar una forma de onda particular formada por dos trenes de pulso consecutivos de una onda cuadrada de cuatro periodos.

Figura 5. Forma de onda

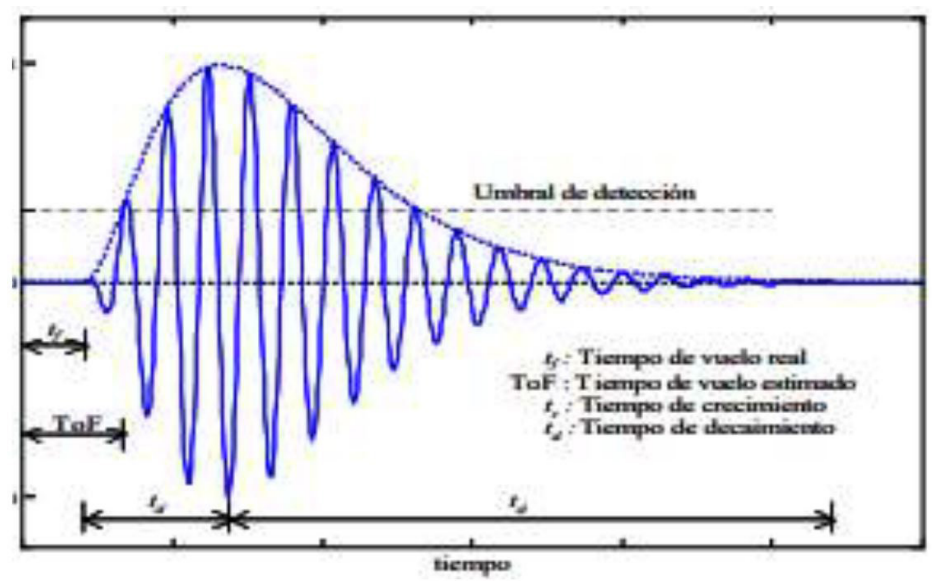

Fuente: elaboración propia. 
2.2. Se implementan tres sensores previamente calibrados en el robot móvil para su posterior automatización.

2.3. Se realizan los cálculos matemáticos y algunas simulaciones para conseguir un rango de error mínimo.

Paso 3. Procesar distancia de las paredes, programación y automatización robot móvil.

3.1. Se procedió a elegir un microcontrolador PIC 16f873A por su tamaño, las salidas que tiene para control y la facilidad de contar con salidas de control PWM, propias de este micro controlador.
3.2. Se usa un lenguaje $\mathrm{C}++$ con un código basado en casos y tareas.

3.3. Se programa el control del robot de manera autónoma con los sensores previamente calibrados.

3.4 Se implementan y se realizan las pruebas en el robot móvil.

Paso 4. Pruebas en pista.

\subsection{Se realizan pruebas en pista.}

4.2. Se realizan los últimos ajustes de software y hardware necesarios.

\section{Resultados}

\section{Partes del robot móvil}

Figura 6. Partes del robot móvil

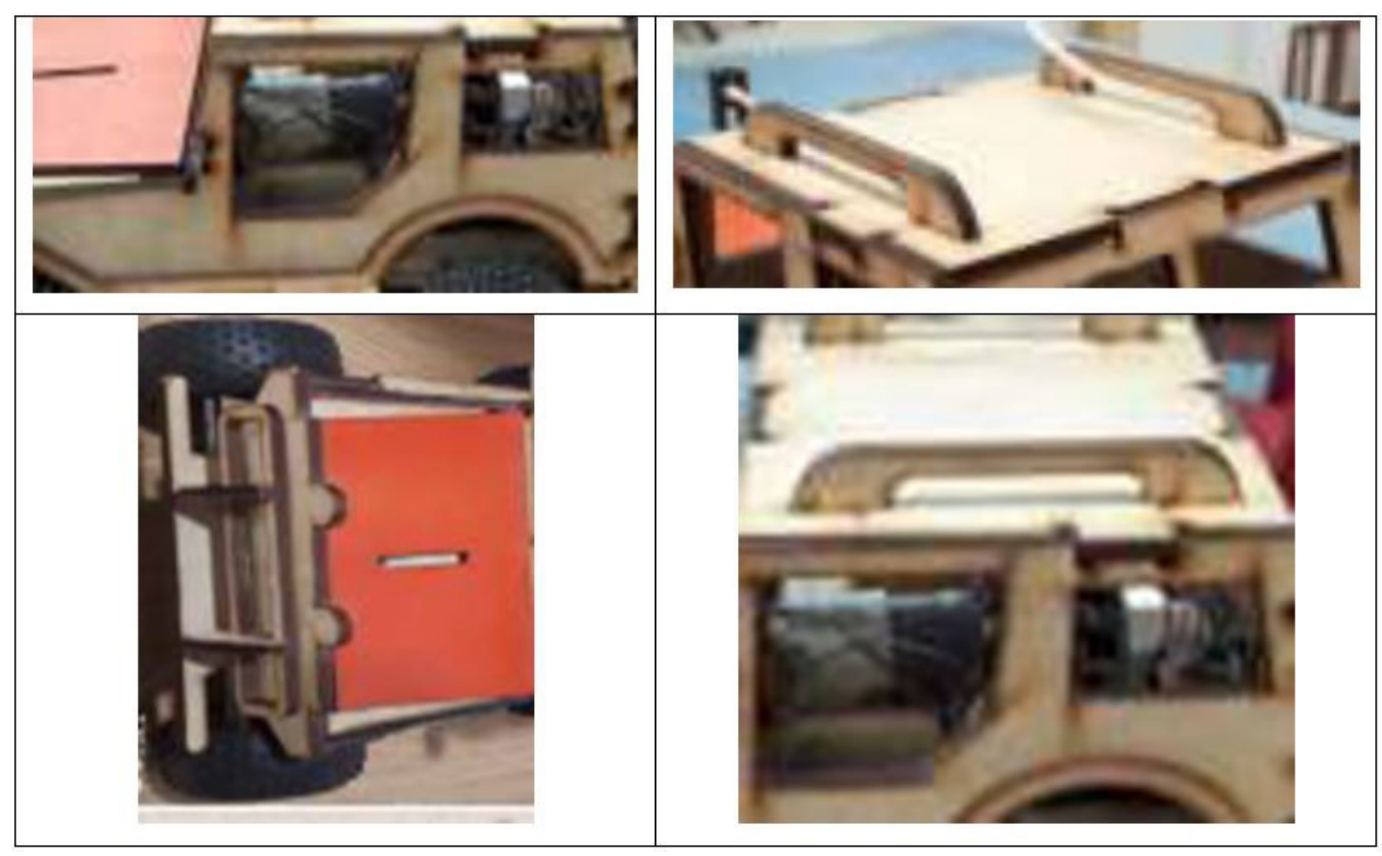




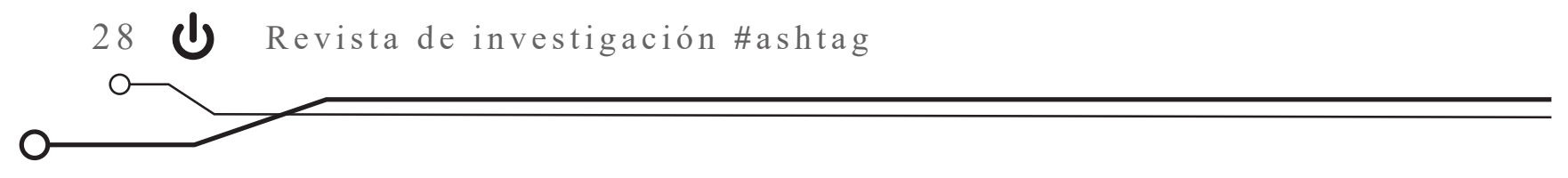

\section{Chasis del robot móvil}

Figura 7. Chasis del robot móvil parte lateral

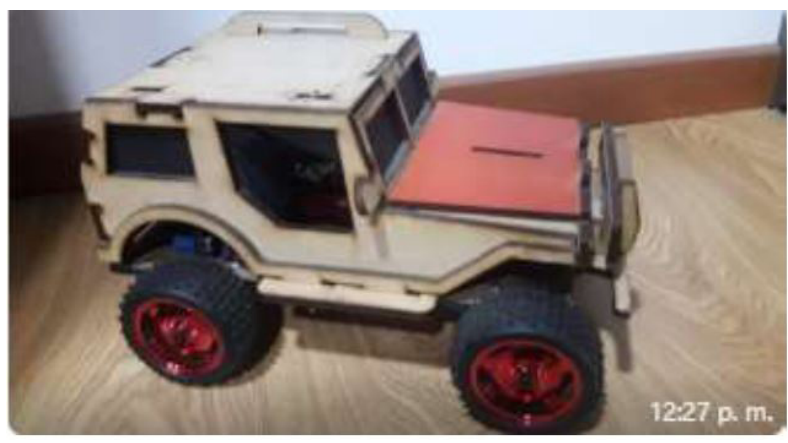

Fuente: elaboración propia.

Figura 8. Chasis del robot móvil parte delantera

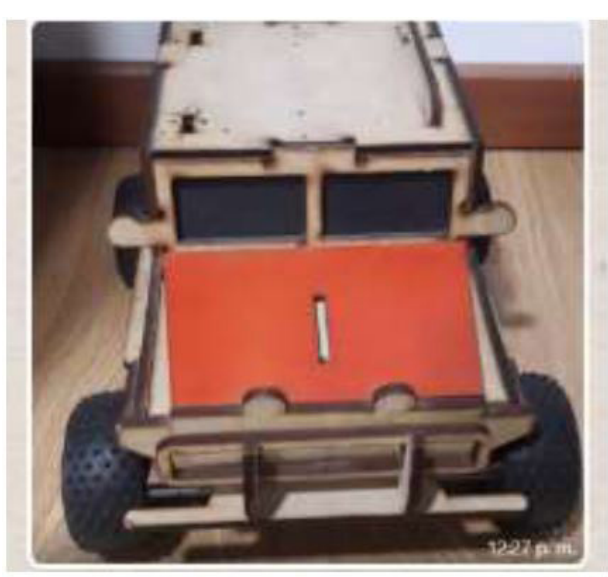

Fuente: elaboración propia.

\section{Sensores calibrados}

Figura 9. Sensores ultrasonido

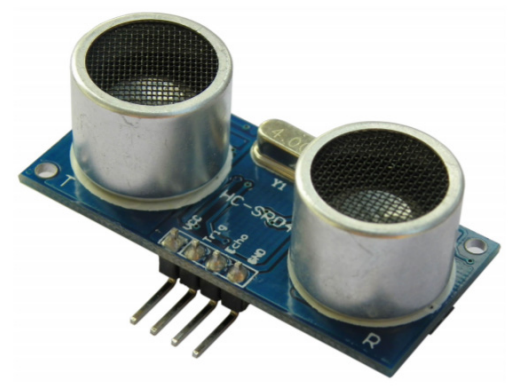

Fuente: Cytron Technologies Sdn. Bhd. (2013) 


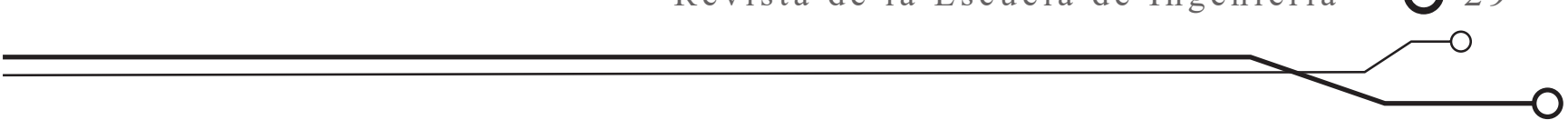

Figura 10. Sensores instalados en el chasis del robot móvil

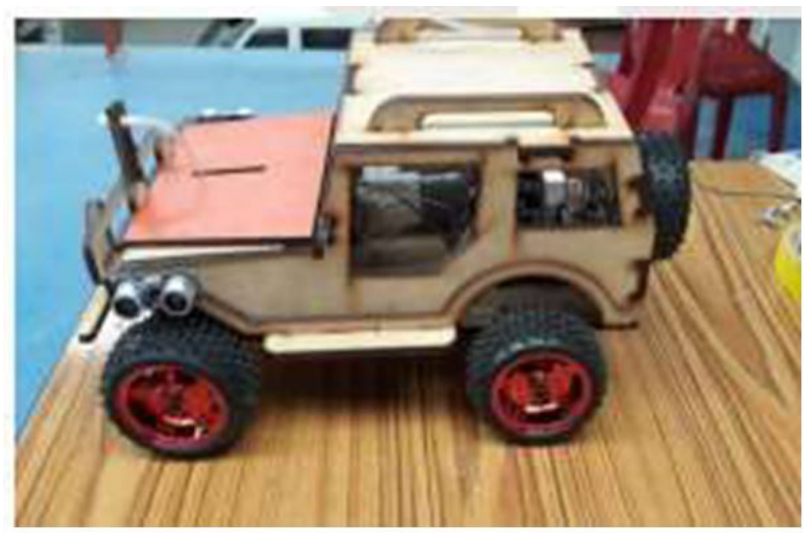

Fuente: elaboración propia.

\section{Motores}

Motores: V=12 V rpm: Fuerza: $2 \mathrm{Kg}$

Encoder 20 huecos

Figura 11. Encoder

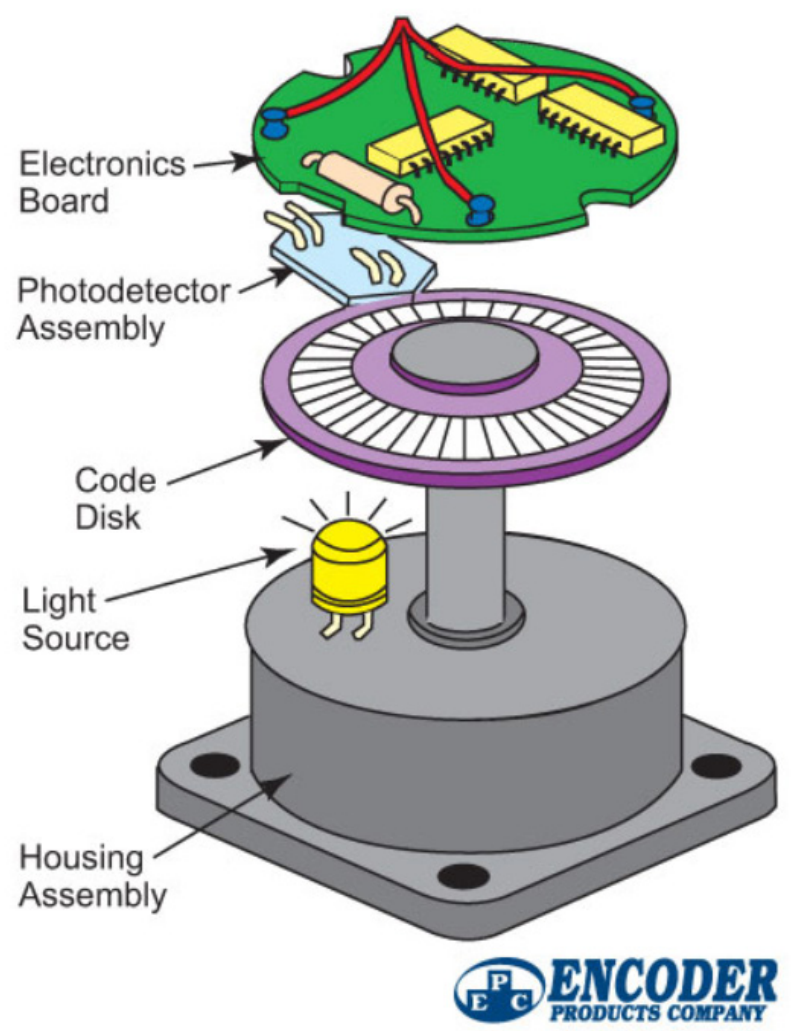

Fuente: Encoder Products Company (2016) 
30 U Revista de investigación \#ashtag

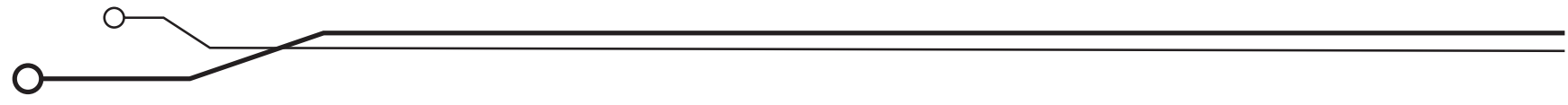

Figura 12. Generador

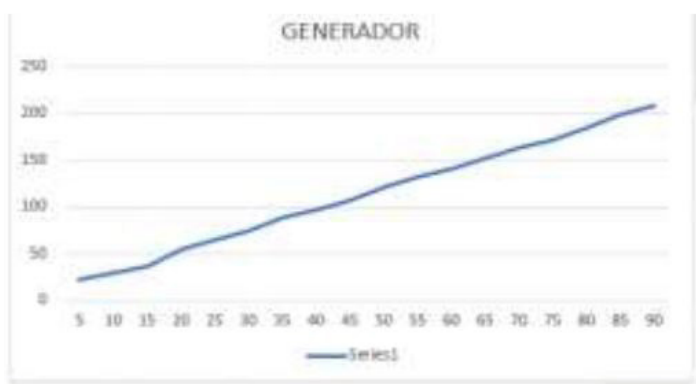

Fuente: elaboración propia.

Figura 13. Tabla de linealidad del encoder

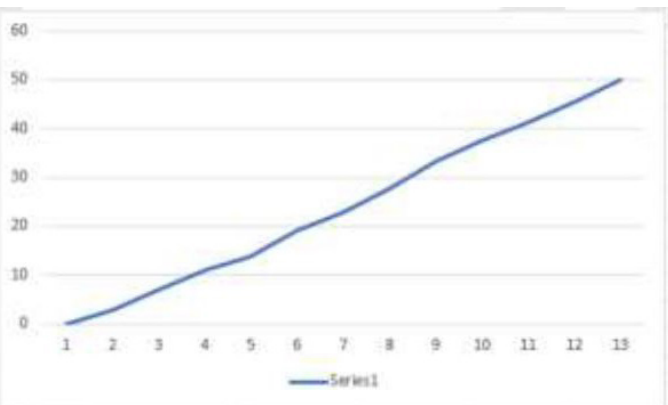

Fuente: elaboración propia.

Tabla 1. Tabla de linealidad del encoder + motor

\begin{tabular}{|c|c|c|}
\hline vol & fre & VRMS (mV) \\
\hline 0 & 0 & 12 \\
\hline 1 & 2,94 & 36,2 \\
\hline 1,5 & 4,16 & 52,5 \\
\hline 2 & 7,14 & 82,8 \\
\hline 2,5 & 10 & 116 \\
\hline 3 & 13,8 & 149 \\
\hline 3,5 & 15,63 & 165 \\
\hline 4 & 17,86 & 195 \\
\hline 4,5 & 20,83 & 242 \\
\hline 5 & 25 & 266 \\
\hline 5,5 & 29,41 & 307 \\
\hline 6 & 33,3 & 336 \\
\hline 6,5 & 35,71 & 364 \\
\hline 7 & 38,46 & 404 \\
\hline 7,5 & 41,67 & 430 \\
\hline 8 & 46,3 & 453 \\
\hline 8,5 & 48,08 & 484 \\
\hline 9 & 51,2 & 502 \\
\hline 9,5 & 52,9 & 515 \\
\hline 10 & 53,19 & 535 \\
\hline
\end{tabular}

Fuente: elaboración propia. 


\section{Resultados en Matlab}

>>núm. $=0.068$

$>>$ den $=[0.212,1]$

$>>\mathrm{G}=\operatorname{tf}($ núm., den) $0.068 \mathrm{G}=0.212 \mathrm{~s}+1$

$>>$ bode $(\mathrm{G})$

Figura 14. Gráficas Matlab
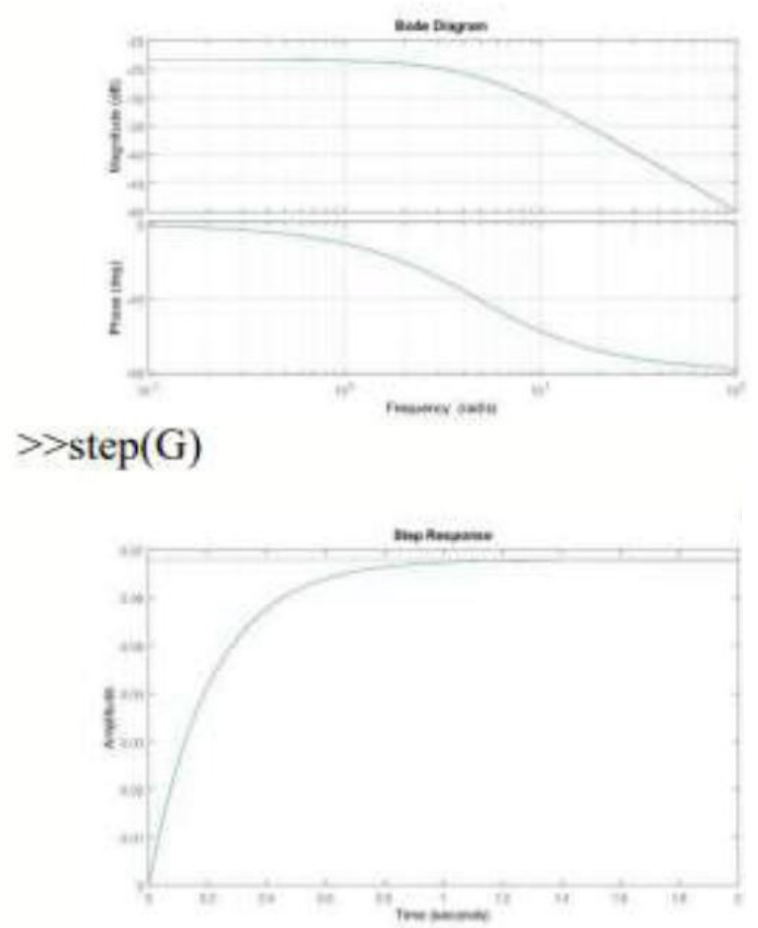

Fuente: elaboración propia.

\section{Implementación del robot}

Figura 15. Implementación del robot

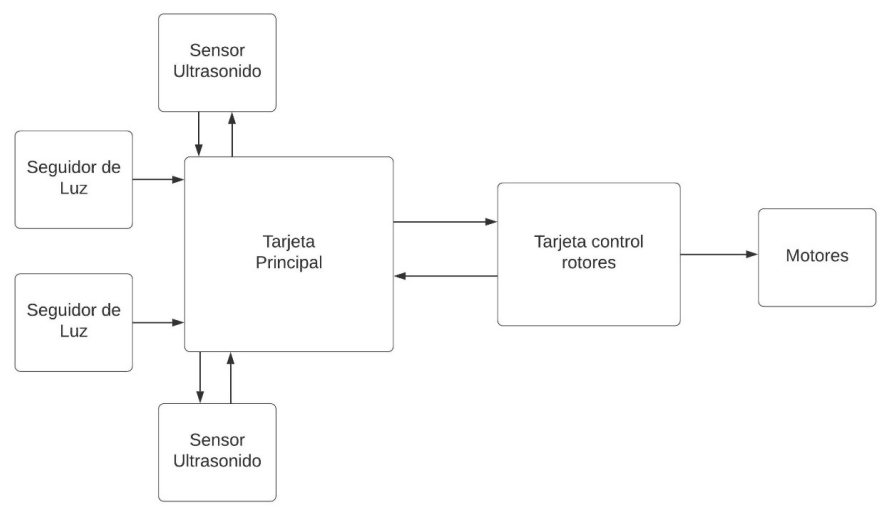




\section{$32 \bigcup$ Revista de investigación \#ashtag}

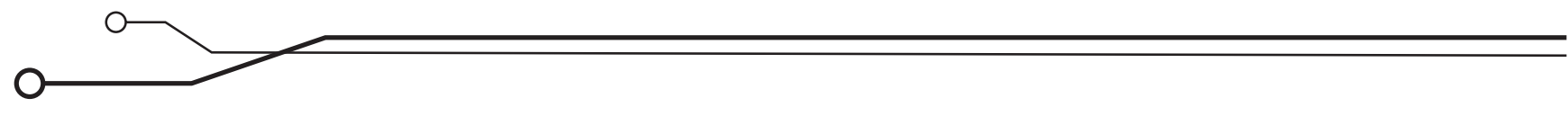

\section{Pruebas en pista}

Figura 16. Robot móvil resultado final

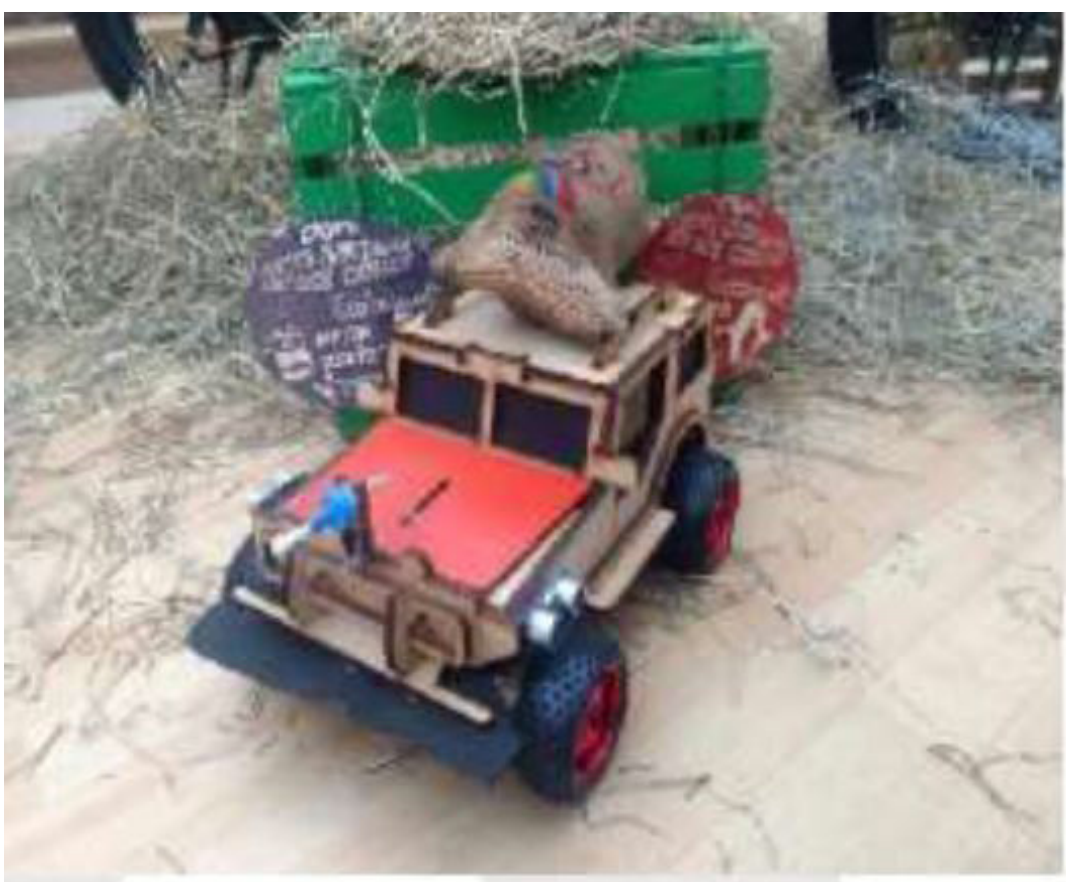

Fuente: elaboración propia.

\section{Conclusiones}

Los sensores de proximidad son elementos electrónicos que varían dependiendo el fabricante y la configuración de fabricación, por lo que es muy importante tener claras las métricas y las dimensiones de lo que se desea sensar con el robot móvil. Se estudió la técnica de medición de distancia por medio de los sensores de ultrasonido y se calculó la distancia por medio del eco, método que dio buenos resultados al momento de pasar el laberinto.

Los artículos similares a nuestro proyecto lograron orientarnos con las ventajas que estos proyectos tenían frente al nuestro $\mathrm{y}$, a la vez, nos permitieron lograr una mejor automatización y un margen de error mínimo. Esta bibliografía también contribuyó a decidir qué sensores se acercaban más a nuestras necesidades.

Los robots móviles en la industria cada vez son más comunes. Básicamente, el principio de todos es muy similar y está basado en robots de poca complejidad que realizamos frecuentemente en nuestros laboratorios. Con la implementación de este proyecto podemos iniciar la creación de robots que puedan realizar tareas mucho más complejas. 


\section{Referencias}

Ackerman, E. (2015). Review: iRobot Roomba 980 - An expensive new Roomba with features and performance to match. IEEE Spectrum. Recuperado de https://spectrum.ieee.org/ automaton/robotics/home-robots/review-irobot-roomba-980

Bambino, I. (2008). Una introducción a los robots móviles. Recuperado de https:/ /n9.cl/f3wlq

Berenz, G. (2008). Mitsubishi RV-M1. Hardware, Software - Proyectos y Soluciones, GIAXWER SOFT. Recuperado de: https://sites.google.com/site/giaxwersoft/mitsubishirobot

Bonilla, M., Quiñones, F., García, I. y Ramírez, J. (2009). Desplazamiento de un robot con localización y evasión de obstáculos por visión y ultrasonido. Congreso Nacional de Ingeniería Electrónica del Golfo Conagolfo 2009. Instituto Tecnológico de Orizaba. Recuperado de https:/ /n9.cl/ c4gdq

Bueno, M. y Ríos, L. (2008). Implementación de comportamientos para navegación inteligente de robots móviles. Tecnura, 11(22), 40-52. Recuperado de https://n9.cl/e1aj

Cytron Technologies Sdn. Bhd. (2013). HC-SR04 Ultrasonic Sensor, User's Manual V1.0. Recuperado de www.datasheetspdf.com/pdf-file/1291829/Cytron/HC-SR04/1

Encoder Products Company (2016). Encoder integration in 2016: New modes of installation, networking, and more. Design World Webinar Series HD. Recuperado de https:// es.slideshare.net/DesignWorldOnline/encoder-integration-in-2016-new-modes-of-installation-networking-and-more

Mendoza, B. (2013). Diseño y construcción del robot móvil "AkuBot" recolector de bolas y desarrollo de laberinto. VIII Olimpiada Nacional de Robótica Universidad de Pamplona 2013, Pamplona, Colombia. Recuperado de https://n9.cl/8rl1k

Millán, R. (2004). Dispositivos de la vivienda domótica. Manual Informativo Acta, 32, 97-101. Recuperado de https:/ / bit.ly/2QH7Uz4

Rodríguez, L. y Reyes, A. (2001). Diseño e implementación de un robot móvil basado en comportamientos. Ciencia, Investigación y Desarrollo, 6(1), 30-33. Recuperado de https:/ / n9.cl/kfy5

Simon, M. (2015). This Incredible Hospital Robot Is Saving Lives. Also, I Hate It. WIRED Magazine. Recuperado de https://www.wired.com/2015/02/incredible-hospital-robotsaving-lives-also-hate/ 